\title{
BMJ Global Health Unethical randomised controlled trial of cervical screening in India: US Freedom of Information Act disclosures
}

\author{
Eric J Suba, ${ }^{1}$ Robert E Ortega, ${ }^{2}$ David G Mutch $^{3}$
}

To cite: Suba EJ, Ortega RE, Mutch DG. Unethical randomised controlled trial of cervical screening in India: US Freedom of Information Act disclosures. BMJ Global Health 2017;2:e000177. doi:10.1136

bmjgh-2016-000177

Received 12 October 2016 Revised 17 February 2017 Accepted 22 February 2017 Published Online First 16 March 2017 tance it was inadvertently published online while awaiting legal review. It has now been withdrawn on legal advice. For the official publisher note please see: http://gh.bmj.com/ content/2/2/bmjgh-2016-000177notice

${ }^{1}$ Kaiser Permanente Medical Center, San Francisco, California, USA

${ }^{2}$ Arizona Republic, Phoenix, Arizona, USA

${ }^{3}$ Washington University School of Medicine, Saint Louis, Missouri, USA 


\section{BMJ Global Health Publisher's Note}

Suba EJ, Ortega RE, Mutch DG. Unethical randomised controlled trial of cervical screening in India: US Freedom of Information Act disclosures. BMJ Global Health 2017;2:e000177. doi: 10.1136/bmjgh-2016-000177.

This paper has been withdrawn. After acceptance it was inadvertently published online while awaiting legal review. It has now been withdrawn on legal advice. 